\title{
Cytokine biomarkers in tear film for primary open-angle glaucoma
}

This article was published in the following Dove Press journal:

Clinical Ophthalmology

22 February 2017

Number of times this article has been viewed

\author{
Divakar Guptal,* \\ Joanne C Wen ${ }^{2, *}$ \\ Janet L Huebner ${ }^{3}$ \\ Sandra Stinnett ${ }^{\prime}$ \\ Virginia B Kraus ${ }^{3,4}$ \\ Henry C Tseng' \\ Molly Walsh'
}

'Department of Ophthalmology, Duke University Medical Center, Durham, NC, ${ }^{2}$ Department of Ophthalmology, University of Washington, Seattle, WA, ${ }^{3}$ Duke Molecular Physiology Institute, ${ }^{4}$ Division of Rheumatology, Department of Medicine, Duke University School of Medicine,

Durham, NC, USA

*These authors contributed equally to this work
Correspondence: Divakar Gupta Department of Ophthalmology, Duke University Medical Center, 235I Erwin Rd, Durham, NC 27703, USA

$\mathrm{Tel}+\mid 9196846611$

Fax +19196818267

Email divakar.gupta@duke.edu
Purpose: To determine the utility of tear film cytokines as biomarkers for early primary open-angle glaucoma (POAG).

Methods: Patients without POAG and eye drop-naïve patients with newly diagnosed POAG were recruited from an academic hospital-based glaucoma practice. Tear films of recruited patients were obtained and analyzed using a multiplex, high-sensitivity electrochemiluminescent enzyme-linked immunosorbent assay for proinflammatory cytokines (IFN $\gamma$, IL-10, IL-12p70, IL-13, IL-1 $\beta$, IL-2, IL-4, IL-6, IL-8, and TNF $\alpha$ ).

Results: Mean concentrations of tear film cytokines were lower in the glaucoma group for 8 of 10 cytokines tested. IL-12p70 (3.94 $\pm 2.19 \mathrm{pg} / \mathrm{mL}$ in control vs $2.31 \pm 1.156 \mathrm{pg} / \mathrm{mL}$ in POAG; $P=0.035$ ) was significantly lower in the tear film of patients with newly diagnosed POAG.

Conclusion: Proinflammatory cytokines were lower in eye drop-naïve newly diagnosed glaucoma patients. Tear film cytokine profiles may be used as biomarkers of early POAG.

Keywords: glaucoma, biomarkers, tear film, cytokines, glaucoma diagnosis, lower limit of detection

\section{Introduction}

Glaucoma is an optic neuropathy that causes visual impairment and blindness through neurodegeneration and apoptosis of retinal ganglion cells. ${ }^{1,2}$ Primary open-angle glaucoma (POAG) accounts for over $70 \%$ of all glaucoma subtypes. ${ }^{3}$ It is estimated that 3.4 million Americans will have POAG by 2020, a 50\% increase from the 2.2 million Americans in $2000 .{ }^{4}$ Over half of these individuals are undiagnosed or untreated. ${ }^{5}$ Current screening techniques have poor sensitivity and are unable to diagnose early POAG. For example, elevated intraocular pressure (IOP) has been used to screen for POAG, but $>50 \%$ of POAG patients have an IOP that is $<21 \mathrm{mmHg} .{ }^{6}$ Screening using automated perimetry for glaucomatous visual field defects lacks the resolution to detect early POAG, as $>35 \%$ of the retinal ganglion cells can be lost before any visual field defects are observed. ${ }^{7}$

Given these limited screening methods, there is a need for new biomarkers of early POAG. Of particular interest is the role of cytokines involved in oxidative stress and inflammation, as these are suspected to have a role in retinal ganglion cell death and the pathogenesis of glaucoma. ${ }^{8}$ A number of studies have identified differential expression of cytokines in the aqueous humor and the anterior chamber tissues of patients with POAG. ${ }^{9-15}$ However, sampling the aqueous humor requires an invasive procedure associated with risks and complications, and thus would be of limited utility as a clinical diagnostic tool. In this study, we have analyzed the tear film of patients with early POAG and healthy control patients. The tear film is easily accessed 
and potentially a novel screening medium for glaucoma diagnosis. The study was performed with newly diagnosed POAG patients who had not yet received any glaucoma eye drops (eye drop-naïve). We hypothesized that tear film cytokines could be utilized as biomarkers for noninvasive and rapid diagnosis of early POAG.

\section{Methods}

\section{Study population}

This study was approved by the Institutional Review Board at Duke University Medical Center and was conducted in accordance with the Declaration of Helsinki. Patients provided written informed consent to participate in the study. Control subjects without eye disease and subjects with a new diagnosis of untreated glaucoma in at least one eye were recruited. Visual acuity, IOP by tonometry and optic nerve head cup-to-disc ratio data were gathered. The diagnostic criteria for glaucoma included the new appearance of general or focal neuroretinal rim thinning, and/or thinning of the retinal nerve fiber layer as seen on optical coherence tomography, and/or a corresponding visual field defect on automated perimetry (Humphrey Field Analyzer II; Carl Zeiss Meditec Inc., Dublin, CA, USA), and open angles by gonioscopy. Diagnosis of glaucoma was made by the subjects' treating ophthalmologist (a fellowship-trained glaucoma specialist). Subjects were characterized as controls if their eyes met none of the diagnostic criteria for glaucomatous changes and had an IOP $\leq 21 \mathrm{mmHg}$. Patients were excluded if they had a history of glaucoma eye drop use within the past 1 month (excluding artificial tears), any contact lens use within the past month, any eye surgery within the past year, and any history of an inflammatory eye condition or diabetes.

\section{Tear collection and protein extraction}

Once informed consent was obtained, tear samples were collected from control and glaucoma patients. Proparacaine was first administered to the eye, and a preweighed Schirmer's tear test strip (Haag-Streit UK Ltd, Harlow, Essex, UK) was placed in the subject's inferior fornix. After 5 minutes, the Schirmer's test strip was again weighed, and the change in weight was used to estimate the volume of collected tears. The test strip was then completely immersed in $70 \mu \mathrm{L}$ of T-PER (Tissue Protein Extraction Reagent; Pierce Chemical, Rockford, IL, USA) within a sterile microcentrifuge tube and soaked overnight at $4^{\circ} \mathrm{C}$. The following day, the Schirmer's tear strip was removed and placed into a second microcentrifuge tube and centrifuged at $>15,000 \times g$ for 2 minutes in a $4^{\circ} \mathrm{C}$ cold room. The liquid from the 2 microcentrifuge tubes was combined, and the sample was stored at $-80^{\circ} \mathrm{C}$ until cytokine analysis. Final tear film cytokine concentrations were determined after correcting for dilutions incurred during testing.

\section{Cytokine analysis}

Thirty-four human tear samples were assayed in singlicate (due to limited sample volumes) using the MSD proinflammatory Panel I, a highly sensitive multiplex enzyme-linked immunosorbent assay (ELISA) for quantitatively measuring 10 cytokines including interferon $\gamma($ IFN- $\gamma)$, interleukin (IL)-1 $\beta$, IL-2, IL-4, IL-6, IL-8, IL-10, IL-12p70, IL-13, and tumor necrosis factor $\alpha$ (TNF $\alpha$ ) from a single small sample volume $(25 \mu \mathrm{L})$ using an electrochemiluminescent detection method (MesoScale Discovery, Gaithersburg, MD, USA). The mean intraassay coefficients based on the standards run in duplicate for each of the cytokines were $<8.5 \%$ for all cytokines (Table 1). For the purposes of statistical analyses, any value that was below the lowest limit of detection (LLOD) for the cytokine assay was replaced with $1 / 2$ LLOD of the assay. This imputation method is robust and wellestablished. ${ }^{16}$

\section{Data analysis}

Descriptive statistics were summarized with means and standard deviations for continuous variables and frequencies and percentages for categorical variables. Comparisons of demographic variables were made using two sample $t$-test for continuous variables or Fisher's exact tests for categorical variables. Comparisons between groups for means of cytokines were carried out using generalized estimating equations to account for the correlation between eyes of

Table I Number and percentage of samples below the LLOD and mean IC

\begin{tabular}{llll}
\hline Cytokine & $<$ LLOD (\%) & IC $\%$ & $<$ LLOD and POAG (\%) \\
\hline IFN $\gamma$ & I I (28.9) & 6.0 & $2(I 2.5)$ \\
IL-I0 & I $3(34.2)$ & 3.8 & $4(25.0)$ \\
IL-I 2p70 & II (28.9) & 8.5 & $3(18.8)$ \\
IL-I3 & I0 (26.3) & 7.7 & $4(25.0)$ \\
IL-I $\beta$ & $0(0.0)$ & 3.0 & $0(0.0)$ \\
IL-2 & $0(0.0)$ & 3.4 & $0(0.0)$ \\
IL-4 & $24(63.2)$ & 7.3 & I0 (62.5) \\
IL-6 & $0(0.0)$ & 4.9 & $0(0)$ \\
IL-8 & $0(0.0)$ & 2.1 & $0(0)$ \\
TNF- $\alpha$ & $0(0.0)$ & 3.2 & $0(0)$ \\
\hline
\end{tabular}

Note: The final column reports the number and percentage of subjects with POAG whose samples were below the LLOD.

Abbreviations: LLOD, lower limit of detection; POAG, primary open-angle glaucoma; IC, intraassay coefficients; IFN $\gamma$, interferon $\gamma$; IL, interleukin; TNF $\alpha$, tumor necrosis factor $\alpha$. 
patients. We analyzed the data using SAS/STAT software (SAS Institute, Inc, Cary, NC, USA). In all cases, a $P$-value $<0.05$ was considered statistically significant.

\section{Results}

Nineteen patients were enrolled in this study, and tear samples were obtained from a total of 38 eyes. Nine patients (18 eyes) were controls, and 10 patients (16 eyes) were diagnosed with early POAG (Table 2). Samples from 4 eyes that were diagnosed as "glaucoma suspects" were not included for analysis in either group. The average age of control patients was $62.4 \pm 8.7$ years, and the average age of POAG patients was $61.5 \pm 15.2$ years $(P=0.949)$. There was no difference in sex between control patients and those with newly diagnosed glaucoma $(P=0.525)$. The control group had a greater proportion of Caucasian patients $(100 \%$ vs $60 \%$ ), though this difference was not statistically significant $(P=0.142$; Table 2$)$.

logMAR visual acuities were similar between the 2 groups: $0.02 \pm 0.09$ in control patients vs $0.17 \pm 0.32$ in patients with POAG $(P=0.094)$. IOP was $14.9 \pm 3.8 \mathrm{mmHg}$ in controls and $17.7 \pm 4.3 \mathrm{mmHg}$ in patients with POAG $(P=0.191)$. Cup-to-disc ratios were significantly different between the 2 groups: $0.31 \pm 0.09$ in controls vs $0.75 \pm 0.15$ in patients with POAG $(P<0.001)$.

Ten cytokines (such as IFN $\gamma$, IL-10, IL-12p70, IL-13, IL-1 $\beta$, IL-2, IL-4, IL-6, IL-8, and TNF $\alpha$ ) were quantified in the tear samples by multiplex ELISA using electrochemiluminescent detection $(\mathrm{pg} / \mathrm{mL}$ ). The range (from minimum to maximum concentrations) for nearly all cytokines spanned 10- to 100-fold in both control and glaucoma groups. In the cases of IFN $\gamma$, IL-10, IL-12p70, IL-13, and IL-4, $>25 \%$ of samples had concentrations that fell below the LLOD of the assay. Concentrations of IL-1 $\beta$, IL-2, IL-6, IL-8, and TNF $\alpha$, however, were all above the LLOD in all samples (Table 1). Mean concentration of 8 or 10 of the cytokines were lower in POAG compared with control patients (Table 1). Mean IL-12p70 cytokine concentrations in tear samples were significantly lower in POAG compared with control patients $(2.31 \pm 1.156 \mathrm{pg} / \mathrm{mL}$ in POAG vs $3.94 \pm 2.19 \mathrm{pg} / \mathrm{mL}$ in controls; $P=0.035$; range of measurable values $0.53-4.74 \mathrm{pg} / \mathrm{mL}$ in POAG vs $1.03-8.67 \mathrm{pg} / \mathrm{mL}$ in control patients). There were no statistically significant differences between groups for the other 9 cytokines (Table 3).

\section{Discussion}

In studies using aqueous humor specimens, cytokine concentration results have been variable in patients with and without glaucoma. In some studies, multiplex cytokine analyses have found several cytokines elevated in open-angle glaucoma. ${ }^{15,17}$ Although other studies have reported lower aqueous IL-6 concentrations in glaucoma, no other cytokines tested were significantly different between patients with glaucoma and controls. ${ }^{11,14}$ These results highlight the complex association of inflammation and glaucoma pathogenesis. ${ }^{8}$ Furthermore, cytokine profiles in the aqueous may change after the use of glaucoma medication, cataract extraction, or selective laser trabeculoplasty, which complicates the interpretation of previous studies. ${ }^{17,18}$ The effect of topical medical therapy on aqueous humor cytokines is not known and could be a potential confounder of cytokine levels in previous studies of patients with chronic glaucoma. ${ }^{19}$

In this study, we analyzed tear film cytokine concentrations. This approach has the advantage that tear samples can be collected less invasively than aqueous humor specimens and therefore represents a more practical screening test. A major strength of this study is that our glaucoma subjects had not been exposed to prior glaucoma eye medications,

Table 2 Demographic and patient characteristic data of control and POAG patients

\begin{tabular}{|c|c|c|c|}
\hline Demographics & Control $(n=9)$, mean \pm SD & POAG $(n=10)$, mean \pm SD & $P$-value \\
\hline Age (years) & $62.4 \pm 8.7$ & $61.5 \pm 15.2$ & $0.949^{a}$ \\
\hline Female $\mathrm{n}(\%)$ & $8(89 \%)$ & $6(60 \%)$ & $0.525^{b}$ \\
\hline \multicolumn{4}{|l|}{ Ethnicity } \\
\hline African American & $0(0 \%)$ & $3(30 \%)$ & $0.142^{\mathrm{b}}$ \\
\hline Caucasian & $9(100 \%)$ & $6(60 \%)$ & \\
\hline Middle Eastern & $0(0 \%)$ & $\mathrm{I}(10 \%)$ & \\
\hline Patient characteristics & Control $(n=18)$, mean \pm SD & POAG $(n=16)$, mean \pm SD & $P$-value ${ }^{c}$ \\
\hline logMAR visual acuity & $0.02 \pm 0.9$ & $0.17 \pm 0.32$ & 0.094 \\
\hline Intraocular pressure & $14.9 \pm 3.8$ & $17.7 \pm 4.3$ & 0.191 \\
\hline Cup-to-disc ratio & $0.3 I \pm 0.9$ & $0.75 \pm 0.15$ & $<0.001 *$ \\
\hline
\end{tabular}

Notes: a $P$-value based on two-tailed $t$-test of difference among means; ${ }^{b} P$-value based on Fisher's exact test of difference among proportions; ${ }^{c} P$-value based on score statistic from GEE analysis of difference between means accounting for correlation between eyes; $* P<0.05$. Bold figure indicates value was $<0.05$.

Abbreviations: POAG, primary open-angle glaucoma; SD, standard deviation; GEE, generalized estimating equations. 
Table 3 Cytokine levels of control and glaucoma POAG patients

\begin{tabular}{|c|c|c|c|}
\hline \multirow[t]{2}{*}{ Cytokine } & \multirow{2}{*}{$\begin{array}{l}\text { Control }(n=18) \\
\text { Mean } \pm \text { SD (range) pg/mL }\end{array}$} & \multirow{2}{*}{$\begin{array}{l}\text { POAG }(n=16) \\
\text { Mean } \pm \text { SD (range) pg/mL }\end{array}$} & \multirow[t]{2}{*}{ P-value* } \\
\hline & & & \\
\hline $\mathrm{IFN} \gamma$ & $16.85 \pm 20.10(1.18-66.83)$ & $14.76 \pm 13.99(2.79-54.72)$ & 0.910 \\
\hline IL-10 & $1.35 \pm 1.69(0.10-5.75)$ & $1.46 \pm 1.73(0.20-7.14)$ & 0.521 \\
\hline IL-I2p70 & $3.94 \pm 2.19(1.03-8.67)$ & $2.31 \pm 1.16(0.53-4.74)$ & 0.035 \\
\hline IL-13 & $30.94 \pm 26.92(2.98-91.39)$ & $18.18 \pm \mid 1.23(3.83-36.46)$ & 0.328 \\
\hline IL-I $\beta$ & $19.22 \pm|6.7|(2.87-60.46)$ & $9.00 \pm 6.08(1.37-23.98)$ & 0.063 \\
\hline IL-2 & $5.06 \pm 4.59(0.85-16.53)$ & $3.38 \pm I .66(I .02-5.8 I)$ & 0.482 \\
\hline IL-4 & $0.38 \pm 0.34(0.06-1.18)$ & $0.25 \pm 0.26(0.03-1.10)$ & 0.162 \\
\hline IL-6 & $86.7 I \pm 88.45(8.07-356.78)$ & $54.62 \pm 67.09(7.39-270.65)$ & 0.173 \\
\hline IL-8 & $4,326.52 \pm 5,766.80(625.19-25,596.07)$ & $2,021.11 \pm 1,584.43(269.25-6,615.59)$ & 0.167 \\
\hline TNF $\alpha$ & $4.86 \pm 2.96(1.89-12.40)$ & $4.91 \pm 1.91(1.19-8.06)$ & 0.727 \\
\hline
\end{tabular}

Notes: $* P$-value based on score statistic from GEE analysis of difference between means accounting for correlation between eyes. The log transformation of cytokines rendered the distributions normal and was used in the analysis. ILI2p70 cytokine values were significantly lower in POAG patients. Bold figure indicates value was $<0.05$. Abbreviations: POAG, primary open-angle glaucoma; IFN $\gamma$, interferon $\gamma$; IL, interleukin; TNF $\alpha$, tumor necrosis factor $\alpha$; SD, standard deviation; GEE, generalized estimating equations.

laser, or ocular surgery, all of which may alter the inflammatory environment of the ocular surface. In chronically medically treated patients with glaucoma, tear cytokine concentrations of monocyte chemoattractant protein 1 have been elevated relative to that in control patients. ${ }^{20}$ Chronically medicated glaucoma patients also demonstrate upregulation of chemokine receptors on the ocular surface and an increase in proinflammatory tear cytokines. ${ }^{21,22}$ Preservatives in ocular drops may also play a role in increasing tear cytokines. ${ }^{23}$

Interestingly, in our study, the assayed cytokines were generally lower in POAG patients, but 1 cytokine in particular, IL-12p70, was significantly lower in POAG patients. IL-12p70 levels must be interpreted with caution as $28.9 \%$ of samples were below the LLOD. In future, we can consider incubating the Schirmer's tear test strip in less T-PER to yield a more concentrated tear sample. However, the fact that tear film concentrations of this cytokine were low in POAG patients is contrary to previous studies that showed higher cytokine concentrations in POAG patients, with preferential increase of cytokines involved in the $\mathrm{T}$ helper cell Type 1 pathway. ${ }^{22}$ A possible explanation for our result may be related to differences of our study population compared with prior studies, as it included only eye drop-naïve patients with newly diagnosed POAG rather than chronically treated glaucoma patients.

The significance of lower cytokines in POAG patients as well as the relationship between cytokines and disease mechanism is unclear. Cytokines may play a role beyond inducing an inflammatory response. For example, in aqueous studies, transforming growth factor $\beta$ was elevated in patients with POAG. ${ }^{14,19}$ Active transforming growth factor $\beta$ was higher in the aqueous of POAG eyes than in eyes with uveitis-related secondary glaucoma. ${ }^{19,24}$ This cytokine has both pro- and anti-inflammatory properties. ${ }^{25}$ This means that a rise of this cytokine in a patient could be explained as worsening disease or be the result of a protective compensatory response by anterior chamber cells. Without longitudinal data, we do not know how cytokines change throughout the course of disease. Cytokines may initially be decreased relative to controls before increasing, or vice versa. Another example of a noninflammatory function for cytokines was observed through IL-1-mediated pathways that were not proinflammatory, but rather a protective response to cellular stress in the anterior chamber of glaucomatous eyes. ${ }^{13}$

There are several limitations of the present study. Although we recruited sufficient numbers of patients to detect statistically significant differences in cytokine expression, a larger sample size would be desirable. Another major limitation was that only a limited number of cytokines were assessed, focusing initially on proinflammatory cytokines, necessitated by the limited sample volumes available for analyses. A third limitation is the current absence of longitudinal data. It would be clinically useful to determine how cytokine expression changes with disease progression or with successful glaucoma therapy. We also do not have data on the variability and reproducibility of this method of sample collection in our study population. Furthermore, although tear sample are easily obtained, they are also exposed to environmental factors and/or ocular surface conditions that could influence and confound cytokine analysis. The effect on assay results due to use of proparacaine, reflex tearing, or collection method (Schirmer's strips) was not investigated. However, we would expect any effect to tear cytokine concentrations due to the collection method to influence 
values of both control and POAG samples. This limitation is less of an issue as we are most interested in the relative values between the 2 groups rather than the absolute concentrations. This study, although small, provides results to calculate the appropriate sample size for adequate powering of a follow-up study.

\section{Conclusion}

Despite the small amount of protein available in a small tear film sample collected from patients, we have demonstrated that it is possible to quantitatively detect protein analytes, such as cytokines. IL-12p70 was statistically lower in eye drop-naïve POAG patients than comparable control patients. Using this novel noninvasive method of sample collection, cytokines and chemokines that are differentially expressed can be identified and utilized to elucidate mechanisms involved in the development and progression of glaucoma, leading to the identification of potential diagnostic biomarkers for POAG.

\section{Acknowledgments}

This study received the Research to Prevent Blindness - 2013 Duke's Unrestricted Grant Award. The funding organization had no role in the design or conduct of this research.

\section{Disclosure}

The authors report no conflicts of interest in this work.

\section{References}

1. Garcia-Valenzuela E, Shareef S, Walsh J, Sharma SC. Programmed cell death of retinal ganglion cells during experimental glaucoma. Exp Eye Res. 1995;61(1):33-44.

2. Quigley HA, Nickells RW, Kerrigan LA, Pease ME, Thibault DJ, Zack DJ. Retinal ganglion cell death in experimental glaucoma and after axotomy occurs by apoptosis. Invest Ophthalmol Vis Sci. 1995;36(5):774-786.

3. Quigley HA, Broman AT. The number of people with glaucoma worldwide in 2010 and 2020. Br J Ophthalmol. 2006;90(3):262-267.

4. Friedman DS, Wolfs RC, O'Colmain BJ, et al. Prevalence of open-angle glaucoma among adults in the United States. Arch Ophthalmol. 2004; 122(4):532-538.

5. Shaikh Y, Yu F, Coleman AL. Burden of undetected and untreated glaucoma in the United States. Am J Ophthalmol. 2014;158(6):1121e1-1129.e1.

6. Sommer A, Tielsch JM, Katz J, et al. Relationship between intraocular pressure and primary open angle glaucoma among white and black Americans. The Baltimore Eye Survey. Arch Ophthalmol. 1991; 109(8):1090-1095.

7. Kerrigan-BaumrindLA, Quigley HA, Pease ME, Kerrigan DF, Mitchell RS. Number of ganglion cells in glaucoma eyes compared with threshold visual field tests in the same persons. Invest Ophthalmol Vis Sci. 2000; 41(3):741-748.
8. Vohra R, Tsai JC, Kolko M. The role of inflammation in the pathogenesis of glaucoma. Surv Ophthalmol. 2013;58(4):311-320.

9. Knepper PA, Mayanil CS, Goossens W, et al. Aqueous humor in primary open-angle glaucoma contains an increased level of CD44S. Invest Ophthalmol Vis Sci. 2002;43(1):133-139.

10. Duan X, Xue P, Wang N, Dong Z, Lu Q, Yang F. Proteomic analysis of aqueous humor from patients with primary open angle glaucoma. Mol Vis. 2010;16:2839-2846.

11. Borkenstein A, Faschinger C, Maier R, et al. Measurement of tumor necrosis factor-alpha, interleukin-6, Fas ligand, interleukin-1alpha, and interleukin-1beta in the aqueous humor of patients with open angle glaucoma using multiplex bead analysis. Mol Vis. 2013;19:2306-2311.

12. Goyal A, Srivastava A, Sihota R, Kaur J. Evaluation of oxidative stress markers in aqueous humor of primary open angle glaucoma and primary angle closure glaucoma patients. Curr Eye Res. 2014;39(8):823-829.

13. Wang N, Chintala SK, Fini ME, Schuman JS. Activation of a tissue-specific stress response in the aqueous outflow pathway of the eye defines the glaucoma disease phenotype. Nat Med. 2001; 7(3):304-309.

14. Takai Y, Tanito M, Ohira A. Multiplex cytokine analysis of aqueous humor in eyes with primary open-angle glaucoma, exfoliation glaucoma, and cataract. Invest Ophthalmol Vis Sci. 2012;53(1):241-247.

15. Chua J, Vania M, Cheung CM, et al. Expression profile of inflammatory cytokines in aqueous from glaucomatous eyes. Mol Vis. 2012;18: 431-438.

16. Vexler A, Tao G, Chen X. A toolkit for clinical statisticians to fix problems based on biomarker measurements subject to instrumental limitations: from repeated measurement techniques to a hybrid pooledunpooled design. Methods Mol Biol. 2015;1208:439-460.

17. Inoue $\mathrm{T}$, Kawaji $\mathrm{T}$, Inatani $\mathrm{M}$, Kameda $\mathrm{T}$, Yoshimura $\mathrm{N}$, Tanihara $\mathrm{H}$ Simultaneous increases in multiple proinflammatory cytokines in the aqueous humor in pseudophakic glaucomatous eyes. J Cataract Refract Surg. 2012;38(8):1389-1397.

18. Alvarado JA, Chau P, Wu J, Juster R, Shifera AS, Geske M. Profiling of cytokines secreted by conventional aqueous outflow pathway endothelial cells activated in vitro and ex vivo with laser irradiation. Invest Ophthalmol Vis Sci. 2015;56(12):7100-7108.

19. Inatani M, Tanihara H, Katsuta H, Honjo M, Kido N, Honda Y. Transforming growth factor-beta 2 levels in aqueous humor of glaucomatous eyes. Graefes Arch Clin Exp Ophthalmol. 2001;239(2):109-113.

20. Chong RS, Jiang YZ, Boey PY, et al. Tear cytokine profile in medicated glaucoma patients: effect of monocyte chemoattractant protein 1 on early posttrabeculectomy outcome. Ophthalmology. 2010;117(12):2353-2358.

21. Baudouin C, Liang H, Hamard P, et al. The ocular surface of glaucoma patients treated over the long term expresses inflammatory markers related to both T-helper 1 and T-helper 2 pathways. Ophthalmology. 2008;115(1):109-115.

22. Malvitte L, Montange T, Vejux A, et al. Measurement of inflammatory cytokines by multicytokine assay in tears of patients with glaucoma topically treated with chronic drugs. Br J Ophthalmol. 2007;91(1): 29-32.

23. Manni G, Centofanti M, Oddone F, Parravano M, Bucci MG Interleukin-1beta tear concentration in glaucomatous and ocular hypertensive patients treated with preservative-free nonselective betablockers. Am J Ophthalmol. 2005;139(1):72-77.

24. Min SH, Lee TI, Chung YS, Kim HK. Transforming growth factor-beta levels in human aqueous humor of glaucomatous, diabetic and uveitic eyes. Korean J Ophthalmol. 2006;20(3):162-165.

25. Sanjabi S, Zenewicz LA, Kamanaka M, Flavell RA. Anti-inflammatory and pro-inflammatory roles of TGF-beta, IL-10, and IL-22 in immunity and autoimmunity. Curr Opin Pharmacol. 2009;9(4):447-453. 


\section{Publish your work in this journal}

Clinical Ophthalmology is an international, peer-reviewed journal covering all subspecialties within ophthalmology. Key topics include: Optometry; Visual science; Pharmacology and drug therapy in eye diseases; Basic Sciences; Primary and Secondary eye care; Patient Safety and Quality of Care Improvements. This journal is indexed on

Submit your manuscript here: http://www.dovepress.com/clinical-ophthalmology-journal
PubMed Central and CAS, and is the official journal of The Society of Clinical Ophthalmology (SCO). The manuscript management system is completely online and includes a very quick and fair peer-review system, which is all easy to use. Visit http://www.dovepress.com/ testimonials.php to read real quotes from published authors. 